Infant Behav Dev. 2013 April ; 36(2): 189-198. doi:10.1016/j.infbeh.2013.01.006.

\title{
Size matters: How age and reaching experiences shape infants' preferences for different sized objects
}

\author{
Klaus Libertus ${ }^{a},{ }^{,}$, Jennifer Gibson ${ }^{b}$, Nadia Z. Hidayatallah ${ }^{c}$, Jane Hirtle ${ }^{d}$, R. Alison Adcock ${ }^{\mathrm{e}}$, \\ and Amy Needham ${ }^{\text {d }}$ \\ aCenter for Autism and Related Disorders, Kennedy Krieger Institute \& Department of Psychiatry \\ and Behavioral Sciences, Johns Hopkins School of Medicine, Baltimore, MD 21211, United \\ States

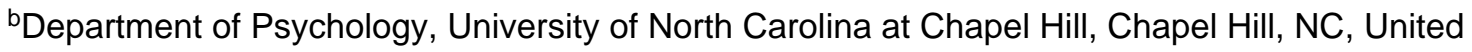 \\ States \\ 'Department of Psychology and Neuroscience, Duke University, Durham, NC, United States \\ dDepartment of Psychology \& Human Development, Peabody College, Vanderbilt University, \\ Nashville, TN, United States \\ eDepartment of Psychiatry and Behavioral Sciences \& Center for Cognitive Neuroscience, Duke \\ University, Durham, NC, United States
}

\begin{abstract}
Looking and reaching preferences for different-sized objects were examined in 4-5- and 5-6month-old infants. Infants were presented with pairs of different sized cylinders and preferences were analyzed by age and reaching status. Outcome variables included looking and touching time for each object, first look, and first touch. Significant three-way interactions with age and reaching status were found for both infants' looking and touching duration. Four-5- and 5-6-month-olds with less reaching experience spent more time visually and manually exploring larger objects. In contrast, 5-6-month-olds with more reaching experience spent more time looking at and touching smaller objects, despite a first look and first touch preference for the largest object. Initially, looking and reaching preferences seem to be driven by mechanisms responding to general visual salience independent of an object's potential for manual action. Once reaching skills emerge, infants begin to use visual information to selectively choose smaller, more graspable objects as exploration targets.
\end{abstract}

\section{Keywords}

Infant; Motor development; Reaching; Preferential looking; Object selection; Grasping

\section{Introduction}

As infants develop new motor skills, opportunities for action emerge and infants begin to detect these opportunities (Gibson, 1988). Reaching is one of the most consequential motor milestones to appear early in life. Reaching allows infants to act on the world, independently obtain and closely examine objects of interest, and engage in triadic social exchanges

\footnotetext{
(c) 2013 Elsevier Inc. All rights reserved.

*Corresponding author. Tel.: +1 443923 7691; fax: +1 443923 7895. klaus.libertus@gmail.com, klaus.libertus@jhu.edu (K. Libertus).
} 
involving objects and people (Bertenthal \& Clifton, 1998; Libertus \& Needham, 2011). Starting at birth, newborns bring their hands toward objects of interest using jerky 'prereaching' movements, often with fisted hands (Campos et al., 2008; von Hofsten, 1982). Reaching with an open hand eventually emerges around 4 months of age and improves steadily over the following months (Pomerleau \& Malcuit, 1980; von Hofsten \& Ronnqvist, 1988). Once an infant engages in reaching, novel opportunities arise for learning about objects, object properties, and the relations between objects (Bourgeois, Khawar, Neal, \& Lockman, 2005; Bushnell \& Boudreau, 1993; Lederman \& Klatzky, 2009; Needham, 2000).

One especially challenging aspect of early motor development is that infants' bodies and abilities are changing rapidly. How responsive are infants' cognitive and motor systems to such changes in strength, size and ability? Do infants alter their actions and goals immediately, once new skills become available? Or do new skills remain unused for weeks or months before infants begin to exploit them?

There is evidence that at least some motor systems - in particular locomotor behavior - are highly responsive to changes in body characteristics. For example, when placed at the top of a steep slope, 14-month-old infants chose to descend while wearing a light (featherweighted) vest and chose not to descend the very same slope while wearing a heavy (leadweighted) vest (Adolph \& Avolio, 2000). Even within the period of a single experimental session, infants' behavior was influenced by their perceived body weight and perhaps the height of their center of mass.

Is there similar evidence for reaching actions being influenced by body characteristics or overall reaching ability and experiences? The answer to this question remains unclear. Bruner and Koslowski (1972) report that 10-week-old infants (too young to be reaching independently yet) flexibly adjust their actions based on the size of the object they are interacting with. Other studies suggest that it is only after infants show independent reaching that they develop the ability to adjust their reaching action prior to object contact (Fagard, 2000; Lockman, Ashmead, \& Bushnell, 1984; Newman, Atkinson, \& Braddick, 2001; von Hofsten \& Ronnqvist, 1988).

\subsection{Factors influencing infants' looking and reaching preferences}

When infants are given two different-sized objects to explore, what factors influence infants' object preferences in such a situation? Do infants start to use information from past reaching experiences to guide their choices soon after they begin reaching, or are more prolonged experiences necessary?

1.1.1. Visual preferences-A number of object-related factors such as size, complexity, and familiarity can influence infants' looking preferences. For instance, newborns prefer to look at the larger of two otherwise identical objects (Slater, Mattock, \& Brown, 1990) and prefer a two-dimensional checkerboard made up of fewer large squares over one made up of smaller squares (Hershenson, 1964). As infants grow older and their visual abilities mature, other variables such as pattern complexity gain more importance in determining visual preferences. While 3-week-old infants prefer to look at checkerboards made up of 4 large squares over those made up of 64 or 576 squares, by 8 weeks infants start to prefer patters of intermediate complexity, and by 14 weeks infants prefer patterns with the highest complexity (i.e., the most squares Brennan, Ames, \& Moore, 1966). Thus with increasing age infants' preference for larger two-dimensional patterns seems to decrease as visual acuity improves and fine details and smaller stimuli are perceived more clearly. Finally, familiarity with a stimulus also influences whether infants will prefer the familiar or a novel stimulus (Hunter, Ames, \& Koopman, 1983). 
Despite the shift from size to complexity, object size remains an important factor influencing infants' visual preferences throughout the first year of life. For example, Newman et al. (2001) showed that infants up to age 12 months first look at larger rather than smaller objects in a preferential-looking paradigm. That infants use size to select objects for closer visual examination is not surprising (Hershenson, 1964; Slater et al., 1990). Larger objects often offer more details to look at and are visually more salient, taking up more of the visual field. However, once objects surpass a certain size they become too large to be grasped and explored manually. Thus, when selecting an object for manual exploration, bigger is not always better.

1.1.2. Manual preferences-Similar to visual preferences, a number of variables can influence infants' reaching and grasping preferences. Newman et al. (2001) examined the influences of object size on reaching preferences in infants aged 5-15 months. In their study, infants were presented with pairs of cylindrical objects of $1 \mathrm{~cm}, 2.5 \mathrm{~cm}$, or $6 \mathrm{~cm}$ in diameter and their visual and reaching preferences were recorded. Between 8.5 and 12 months, infants were significantly more likely to reach for smaller, more graspable objects and to look back and forth between the objects more, suggesting active comparison of the objects before reaching. In contrast, infants aged 5-8.5 months showed no clear reaching preference for either smaller or larger objects, although these infants consistently choose to look at the largest object first. These results show that by 8.5 months of age, but not before, infants begin to make use of object size to guide their manual actions. Perceived reachability also influences infants' reaching for objects. Infants old enough to reach independently for objects will do so primarily when they perceive the objects to be within reach (Rochat \& Goubet, 1995; Yonas, Elieff, \& Arterberry, 2002; Yonas \& Hartman, 1993).

\subsection{The current study}

Few studies have utilized a combined preferential-looking and preferential-reaching paradigm to investigate the relation between factors attracting visual attention and factors encouraging manual exploration. However, this question is important because infants may look and reach toward objects for different reasons. Larger objects, for instance, may be visually appealing but are more unwieldy to hold, too big for oral exploration, and difficult to explore manually. The present study aims to determine whether infants become motivated to reach for objects small enough to grasp once they begin independent reaching for objects. Our study examines these preferences in infants between 4 and 5 months of age and explicitly incorporates reaching ability to assess the influences of object size and reaching success on infants' preferences.

On average, the frequency of reaching is significantly lower in 4-month-olds than in 5month-olds (Rocha, Silva, \&Tudella, 2006). By 5 months of age, infants have probably had several experiences with successful reaching and object manipulation. In contrast, 4-monthold infants most likely have had only minimal reaching experiences or no reaching experiences at all. To capture these experiential differences, infants at each age were divided into more-skilled reachers and less-skilled reachers based on the frequency of their own reaching behaviors during our study. It was hypothesized that younger infants and those less skilled at reaching would attend to and reach for larger, visually salient objects. In contrast, older infants and those more skilled at reaching would make more use of information regarding an object's potential for manual exploration and grasping. Consequently, we hypothesize that more-skilled 5-6-month-olds will attend to and reach for smaller, easily graspable objects when given a choice between two different sized objects. Such findings would indicate that infants' use information from first-hand reaching experiences to guide future actions, overriding visual salience-driven processes that are in place prior to the onset of successful reaching. 


\section{Method}

\subsection{Participants}

A total of 32 infants participated in this study. Participants were divided into two groups based on age: a group of 4-5-month-olds ( $n=14,7$ females, mean age $=137.4$ days, range 125-147 days); and a group of 5-6-month-olds ( $n=18,8$ females, mean age $=168.9$ days, range 156-188 days). All participants were recruited from public birth records in the Research Triangle area of North Carolina. The Duke University IRB approved this study and written informed consent was obtained from a parent or guardian prior to participation of their child. Each family received $\$ 5$ travel reimbursement for their participation.

\subsection{Apparatus}

Four cylindrical shaped canisters, made out of transparent plastic, were used as stimuli. The canisters were closed on top and did not offer any handles of loops for easy grasping. The stimuli varied systematically in size and are labeled objects $1-4$ in our analyses. The objects were $5 \mathrm{~cm}$ in diameter $\times 3 \mathrm{~cm}$ in height (object 1), $7 \mathrm{~cm}$ in diameter $\times 5 \mathrm{~cm}$ in height (object 2), $12 \mathrm{~cm}$ in diameter $\times 13 \mathrm{~cm}$ in height (object 3), and $18 \mathrm{~cm}$ in diameter $\times 24 \mathrm{~cm}$ in height (object 4). Strips of blue, green, red, and white tape were attached around the width of the canisters to make them more visually appealing (see Fig. 1). Infants were seated across from the experimenter on the parent's lap at a table with a half-circle (radius $23 \mathrm{~cm}$ ) cut out on the infants' side. Stimuli were presented in pairs on a rectangular foam core tray, approximately $45 \mathrm{~cm}$ apart from each other. A non-slip surface was attached to the tray so that objects would not accidentally move, but the objects themselves were not attached to the tray. Infants were allowed to freely manipulate and move the objects around during a trial. The experimenter intervened only if an object rolled out of reach or fell off the table, in such circumstance the experimenter replaced the object on its original position on the tray.

\subsection{Procedure}

A combined preferential-looking, preferential-reaching paradigm was used in which infants were repeatedly presented with pairs of objects in a counterbalanced order and position (see below). Front surfaces of both objects were equidistant from the infant throughout the presentation. Each object pair was first presented clearly out of reach for the infant (far position) and then moved by the experimenter to a much closer position, clearly within reach for the infant (near position). Both positions were marked on the table and were the same for all infants. To avoid parent or experimenter induced bias on infants' behavior, parents were asked to refrain from drawing attention to any of the objects and the experimenter looked down throughout the experiment.

During the study, the experimenter wore yellow rubber gloves in order to prevent the objects from slipping out of the experimenters' hand. The experimenter grasped and lifted all objects from the top using one hand and presented two objects simultaneously to the infant (one in each hand). Occasionally pairs involving object 4 were presented sequentially due to the large size of this object. In this case, the order in which objects were presented was counterbalanced across trials.

2.3.1. Warm-up trials-Two warm-up trials were completed prior to the experiment using sets of identical rubber cubes $(6 \mathrm{~cm} \times 6 \mathrm{~cm})$ to familiarize the infant with the experimental procedure. During warm-up trials, two cubes were first presented in the far position for $10 \mathrm{~s}$, and then moved simultaneously to the near position for $20 \mathrm{~s}$. This procedure was repeated with the second set of cubes. 
2.3.2. Test trials-Test trials began immediately following the warm-up trials. During test, pairs of different sized objects were presented first in the far position for $10 \mathrm{~s}$, then moved to the near position for $20 \mathrm{~s}$. A total of 12 test trials with 6 unique object combinations were completed. Two pre-defined conditions were used to ensure that each participant was presented with every possible combination of objects twice and that no object was presented on the same side across two consecutive trials. In condition 1 (18 participants), trials proceeded in the following order (left digit represents the object presented on the experimenter's left (infant's right), right digit represents object on experimenter's right): $1-4,3-2,2-1,4-3,3-1,2-4,4-1,2-3,1-2,3-4,1-3,4-2$. Condition 2 (14 participants) was identical to condition 1 but with the opposite object arrangements: 4-1, 2-3,1-2, 3-4,1-3, 4-2,1-4, 3-2, 2-1, 4-3, 3-1, 2-4. Completing all 12 trials, participants saw each of the 4 objects a total of 6 times in 3 unique combinations. If an infant was not interested in either object on a given trial, the experimenter drew attention to the objects by briefly tapping the top of both objects simultaneously. At the end of each trial, the experimenter retrieved both objects and moved them out of sight.

Ten participants did not complete all 12 trials due to fussiness and crying. However, all completed the first six trials and saw each object pair at least once. Average looking and touching durations for each object were calculated across all completed trials of a particular participant.

Looking and touching behavior was coded offline from video recordings using a real-time coding software (accuracy 1/10 s) to capture behavioral dynamics. Real-time coding has the advantage that it provides an immediate context for each behavior. However, short glances, and brief hand actions may be missed using this approach. To determine reliability of the coding, different observers recoded $94 \%$ of all videos using a frame-by-frame coding program that is better in detecting short glances and brief actions (Libertus, 2008). Overall correlations between the two coding methods were excellent (Looking: $r=.92$; Touching: $r$ $=.97$ ) and are in agreement with previous reports using a similar approach (Libertus \& Needham, 2010). In the following, all analyses are based on the real-time coding results because frame-by-frame coding was not possible for two participants whose video recordings were inadvertently destroyed following real-time coding.

The primary coding using real-time coding software also allowed us to identify the targets of infants' first look and first touch during a trial. Reliability for infants' first look and first touch was separately established by a second observer coding 14 randomly selected participants (44\% of the sample). The two observers agreed on 12 out of 14 first look choices (86\% agreement) and on 14 out of 14 first touch choices (100\% agreement). For the two videos where the observers disagreed, a third observer reviewed the clips and a consensus was reached.

2.3.3. Dependent variables-Dependent variables were overall looking and touching durations. Looking duration was defined as the amount of time an infant spent looking at an object during each trial in which this object was involved. Observers coded every look, including short glances at the objects. Similarly, touching time was the amount of time an infant spent in physical contact with an object using either hand during each trial in which this object was involved. If both hands were used during a touch episode, the touch episode started with the first contact of either hand and ended when there was no more touching by either hand. In addition to overall looking and touching durations, infants' first look and first touch choice regardless of total duration were also analyzed. 


\subsection{Reaching status}

It was assumed that infants who were more successful in contacting the objects were better at organizing their reaching actions. The mean touching duration across all participants was $9.4 \mathrm{~s}$ with a range of $0-21.7 \mathrm{~s}$. Seventeen infants spent less than $8 \mathrm{~s}$ per trial touching the objects and were categorized as 'less-skilled reachers'. The remaining 15 infants spent more than $10 \mathrm{~s}$ per trial touching the objects and were categorized as 'more-skilled reachers'. It is highly likely that these infants behaved in a similar fashion at their own homes and that lessskilled reachers would not have much first-hand reaching experiences at home, whereas more-skilled reachers were likely to engage in reaching more routinely. Once reaching status was assigned, infants were grouped into 4-5-month-old less-skilled reachers $(n=11, M=$ 136.3 days, $\mathrm{SD}=7.41), 4-5$-month-old more-skilled reachers $(n=3, M=141$ days, $\mathrm{SD}=$ 1.00), 5-6-month-old less-skilled reachers ( $n=6, M=168.5$ days, $\mathrm{SD}=11.36)$, and 5-6month-old more-skilled reachers $(n=12, M=169.1, \mathrm{SD}=9.29)$. Because group memberships were not experimentally determined or randomized, highly unequal group sizes were unavoidable. Indeed, uneven distributions in reaching status are to be expected at the ages tested. This limitation should be considered when interpreting our results, in particular with regard to the 4-5-month-old more-skilled reachers.

\section{Results}

\subsection{Four object comparisons}

In a first set of analyses, we compared infants' looking and touching behavior across all four objects. The goal of these analyses was to identify whether infants have a visual or manual preference for one of our four objects over the others and whether this preference is related to reaching status and age.

3.1.1. Looking preferences-An Object $(4) \times$ Age $(2) \times$ Reaching Status $(2) \times$ Gender (2) repeated measures ANOVA with Greenhouse-Geisser correction (for a violation of sphericity, Mauchly's W(5) $=.50, p<.01)$ revealed a significant main effect of Object $(F(2$, $48.21)=6.57, p<.01)$, a significant effect of Reaching Status $(F(1,12)=13.90, p<.01)$, and a significant Object by Age interaction $(F(2,48.21)=4.88, p=.01)$. These effects were qualified by a significant Object by Age by Reaching Status three-way interaction $(F(2$, $48.21)=4.09, p=.02)$. No other effects were significant and gender was excluded as a factor in all subsequent analyses.

The significant three-way interaction was investigated further by conducting separate Object (4) $\times$ Age (2) repeated measures ANOVAs with the less-skilled reachers and the moreskilled reachers respectively. For the less-skilled reaching group $(n=17)$, the ANOVA yielded a significant effect of Object $(F(3,45)=4.92, p<.01)$ but no other effects or interactions (all $p s>.3$ ). Follow-up pairwise comparisons (Bonferroni corrected) between the four objects revealed that the less-skilled reaching infants looked significantly longer at the largest object $(M=7.60, \mathrm{SD}=3.78)$ compared to the smallest object $(M=4.09, \mathrm{SD}=$ $3.72 ; t(16)=3.62, p=.01)$. No other comparisons were significant (all $p s>.09)$.

For the more-skilled reaching group $(n=15)$, the ANOVA revealed a significant effect of Object $(F(3,39)=2.96, p=.04)$ and a significant Object by Age interaction $(F(3,39)=7.11$, $p<.01)$ but no significant effect of Age $(p=.25)$. Follow-up pairwise comparisons (Bonferroni corrected) between the four objects were conducted separately for 4-5 and 5-6month-old infants. More-skilled reaching 4-5-month-old infants $(n=3)$ looked significantly longer at the largest $(M=14.55, \mathrm{SD}=3.29)$ compared to the smallest object $(M=6.28, \mathrm{SD}$ $=2.42 ; t(2)=16.37, p=.02)$, In contrast, more-skilled reaching 5-6-month-old infants $(n=$ 12) showed no differences in looking durations across objects (all $p s>.7$ ). Thus, the Object 
by Age interaction was driven by more-skilled reaching 4-5-month-old infants showing less visual interest in the smallest object and more visual interest in the largest object, whereas the more-skilled reaching 5-6-month-olds showed about equal looking at all four objects (see Fig. 2). Finally, looking duration to the smallest object was additionally compared between the more-skilled reaching 5-6-month-olds $(n=12)$ and all 4-5-month-old infants combined $(n=14)$. This planned comparison revealed that more-skilled-reaching 5-6month-olds looked significantly longer at the smallest object $(M=11.36, \mathrm{SD}=2.24)$ compared to $4-5$-month-old infants regardless of reaching status $(M=4.80, \mathrm{SD}=3.53 ; t(24)$ $=5.55, p<.01)$.

In summary, infants' looking preferences suggest that more-skilled reaching 5-6-month-old infants looked more toward the smallest, most graspable object compared to 4-5-month-old infants whose attention was mostly drawn to the largest, most visually salient object. These findings suggest that infants' visual preferences are influenced by both age and first-hand reaching experiences.

3.1.2. Reaching preferences-An Object $(4) \times$ Age $(2) \times$ Reaching Status $(2) \times$ Gender (2) repeated measures ANOVA with Greenhouse-Geisser correction (for a violation of sphericity, Mauchly's $W(5)=.48, p<.01)$ revealed a significant main effect of Reaching Status $(F(1,24)=71.06, p<.01)$ and an Object by Age interaction $(F(2.14,51.25)=3.19, p$ $=.046$ ). Both effects were qualified by a significant Object by Age by Reaching Status three-way interaction $(F(2.14,51.25)=6.54, p<.01)$. No other effects were significant and gender was excluded as a factor in all subsequent analyses.

The significant three-way interaction was investigated further by conducting two separate Object (4) $\times$ Age (2) repeated measures ANOVAs for the less-skilled reachers and the moreskilled reachers respectively. For the less-skilled reachers $(n=17)$, the ANOVA with Greenhouse-Geisser correction (for violation of sphericity, $W(5)=.31, p<.01$ ) yielded no significant effects or interactions. Although the effect of Object $(F(1.71,25.67)=3.23, p=$. 06) approached significance, follow-up pairwise comparisons (Bonferroni corrected) did not indicate differences in touching duration between the four objects (all $p s>.2$ ).

For the more-skilled reaching group $(n=15)$, the ANOVA revealed a significant Object by Age interaction $(F(3,39)=5.15, p<.01)$. Follow-up pairwise comparisons (Bonferroni corrected) between the four objects were conducted separately for 4-5 and 5-6-month-old infants. More-skilled reaching 4-5-month-old infants $(n=3)$ touched the largest object $(M=$ $9.53, \mathrm{SD}=2.03)$ significantly longer than the smallest object $(M=1.48, \mathrm{SD}=1.74 ; t(2)=$ $16.31, p=.02)$. In contrast, more-skilled reaching 5-6-month-old infants $(n=12)$ showed no differences in their touching durations, regardless of object size (all $p \mathrm{~s}>.7$ ). Thus, the Object by Age interaction was driven by the more-skilled reaching 4-5-month-old infants showing less touching of the smallest object and more touching of the largest object, whereas the more-skilled reaching 5-6-month-olds show about equal touching of all objects (see Fig. 3). Finally, touching of the smallest object was additionally compared between the more-skilled reaching 5-6-month-olds $(n=12)$ and all 4-5-month-old infants combined $(n=$ 14). This planned comparison revealed that 5-6-month-old more-skilled reachers touched the smallest object $(M=10.80, \mathrm{SD}=4.40)$ significantly longer than 4-6-month-old infants regardless of reaching status $(M=.92, \mathrm{SD}=1.45 ; t(13.04)=7.44, p<.01)$. In summary, infants' touching preferences suggest that more-skilled reaching 5-6-month-old infants engage in more touching of the smallest, most graspable object compared to 4-5-month-old infants. Together with the results on infants' visual preferences, this pattern supports the claim that age and reaching experience interact to influence infants' visual attention toward and manual engagement with objects. 


\subsection{Smallest vs. largest object: first look and first touch}

Infants' looking and touching behavior was examined in more detail on the first trial where object 1 (smallest) and object 4 (largest) were paired. Absolute and relative size differences were greatest between these two objects (differing by a 1:3.6 ratio in diameter), and looking and touching differences were most pronounced comparing object 1 to object 4 (Figs. 2 and 3 ). Contrasting object 1 with object 4 allows us to directly examine the competing roles of visual attraction (salient, large object) and object affordances (graspable, small object) during infants' interactions with these objects.

Across all groups, a majority of infants showed a strong tendency to first look at the largest object. A total of 23 out of 32 infants first looked at the largest object $(p<.01$, binomial procedure).This was the case for 9 out of 11 less-skilled reachers at age 4-5 months and for 1 out of 3 more-skilled reachers at age 4-5 months. Similarly, at age 5-6 months 4 out of 6 less-skilled reachers and 9 out of 12 more-skilled reachers showed this preference.

Touching preferences showed a very similar pattern, but could not be analyzed for 7 infants who did not touch either object during the trial (5 less-skilled reachers from the 4-5-monthold group, and 2 less-skilled reachers from the 5-6-month-old group). Overall, the majority of infants who did touch the objects showed a preference to touch the largest object first (17 out of 25 infants, $p=.03$, binomial procedure). At age $4-5$ months, this was the case for 5 out of 6 less-skilled reaching infants, and for 1 out of 3 more-skilled reaching infants. At age 5-6 months, this was the case for 2 out of 4 less-skilled reaching infants, and for 9 out of 12 more-skilled reaching infants. Thus, even the more-skilled reaching 5-6-month-olds who showed an increasing visual and manual preference for the smallest object, still exhibited an initial visual and manual preference for the largest, most visually salient object. It is likely that this preference for larger objects decreases as infants gain more reaching experience (Newman et al, 2001).

\section{Discussion}

The goal of the current study was to investigate how infants' age and motor abilities influence their choices for visual and manual exploration of objects. The question addressed here was whether infants' own developing proficiency with reaching and grasping actions would affect how they direct their visual and manual attention toward different sized objects. This question was examined in infants who are in the process of making the transition into independent reaching.

\subsection{Visual preferences}

In agreement with previous findings, our results indicate that infants who are less skilled at reaching prefer looking at larger objects rather than smaller objects (Hershenson, 1964; Slater et al., 1990). More-skilled reaching 4-5-month-olds also prefer larger over smaller objects, whereas more-skilled reaching 5-6-month-old infants show no preference for larger over smaller objects. When compared to all 4-5-month-olds combined regardless of reaching status, more-skilled reaching 5-6-month-olds show significantly more interest in the smaller, easily graspable objects.

Our results confirm our original hypothesis that less-skilled reaching infants would look more at larger compared to smaller objects. More-skilled reaching 5-6-month-old infants did not show such a pattern and attended to and acted on both objects about equally. However, across both age and skill groups, initial visual preferences were controlled by visual saliency and not by considerations about an objects' graspability (or 'selection-foraction').This pattern of results is consistent with the idea that initial visual preferences (or the infants' first orienting response) are driven by more automatic, bottom-up processes, 
whereas subsequent selection-for-action processes are based on slower, top-down processes. As infants gain more reaching experience, their reaching may become more automatic and less dependent on top-down processes (Bushnell, 1985).

\subsection{Manual preferences}

Results for infants' overall touching durations mirror those of their overall looking preferences, indicating a tight coupling in infants visual and manual choices. Again, 4-5month-old infants and less-skilled reaching 5-6-month-olds spent more time touching the larger objects than the smaller ones. In contrast, the more-skilled reaching 5-6-month-old infants touched all objects about equally regardless of size, but showed significantly stronger interest in the smallest object when compared to all 4-5-month-old infants combined.

\subsection{Deciding what to reach for}

Infants' decisions about whether to try to reach for an object, and if so, which object to reach for, are influenced by their physical abilities. If they cannot yet reach independently, infants are obviously not concerned with factors influencing the reachability of an object (e.g., size, distance). Once independent reaching emerges, infants' choices are now motivated by different factors, including opportunities for manual (and not just visual) exploration of objects. This process may be akin to metamemory in older children (Pressley, Borkowski, \& O'Sullivan, 1984), which helps children decide when to employ memory strategies to help them remember items. In that case, young children must understand when they may have difficulty remembering something (for instance, when the list of to-be-remembered items is quite long) and use a strategy (such as rehearsal) to help them remember. Here, infants need to determine whether to engage in reaching behavior or not, and factors such as the size of the object and the perceived distance to the object may contribute to this decision. Our results suggest that infants incorporate knowledge about object size relatively soon after reaching begins. As in the development of locomotion, infants incorporate information about their changing bodies into their action systems quite soon after these changes occur (Adolph \& Avolio, 2000).

\subsection{Reaching experiences and object choice}

Our results confirm that large objects strongly attract the visual attention of 4-5- and 5-6month-old infants. But once infants have finished their initial visual and manual exploration of an object, their preferences may change depending on the amount of first-hand reaching experiences and age. While both 4-5-month-olds and 5-6-month-old less-skilled reachers continue to attend to and act on larger objects, 5-6-month-olds who are more skilled at reaching become more interested in smaller, more graspable objects. Though infants as young as 4 months of age seem to be sensitive to the graspability of objects and adapt their grip configurations when presented with objects of different sizes (Newell, Scully, McDonald, \&Baillargeon, 1989; Rocha et al., 2006), at this age infants may not yet be able to make choices based on the relative graspability of two simultaneously presented objects.

Five-to-six-month-old more-skilled reachers have arguably spent more time manually exploring and examining objects in their environment. By this age, infants seem to make use of their own experiences to discriminate between an object that is visually appealing and one that is manually appealing. Nevertheless, 5-6-month-old more-skilled reachers still choose to first look at and touch larger objects, suggesting that the ability to judge graspability is still emerging at this age. This pattern also supports the claim that while younger infants may reach reflexively to the first object on which they fixate, older infants seem to analyze relevant object features, including size, and reach for the object with which it will be most rewarding to manually interact (Newman et al., 2001). 
A number of studies now support the view that infants' own experiences and motor skills influence their engagement with objects. For example, studies indicate that providing novel motor experiences such as reaching, postural, or locomotor experiences can facilitate infant's engagement with objects, their information processing, causal perception, and mental rotation skills (Libertus \& Needham, 2010, 2011; Lobo \& Galloway, 2008; Lobo, Galloway, \& Savelsbergh, 2004; Rakison \& Krogh, 2012; Schwarzer, Freitag, Buckel, $\&$ Lofruthe, 2013). Similarly, studies show that infants use information about object distance (Yonas \& Hartman, 1993) and their own postural control skills (Rochat, Goubet, \& Senders, 1999) to determine whether an object affords reaching or not. The current findings add to this literature and suggest that infants start to use relative object size to determine graspability once they have obtained relevant reaching experiences around 5 months of age.

\section{Limitations and future studies}

The results of the present study are consistent with the overall findings by Newman et al. (2001) that infants initially prefer to look first at larger objects and with increasing age start to reach more frequently for smaller, more graspable objects. Newman et al. (2001) tested infants between 5 and 15 months of age but did not find evidence for an effect of object size on reaching in 5- to $81 / 2$-month-old infants. In contrast, we did observe sensitivity to object size already in 5-6-month-old infants with sufficient prior first-hand reaching experiences. At the same time, our negative results in 4-5-month-olds stand in contrast to positive results with 3-month-old infants by Bruner and Koslowski (1972). Absolute object size may offer one explanation for the discrepant findings among these three studies. Bruner and Koslowski (1972) used rather large objects with a big relative size difference $(3.18 \mathrm{~cm}$ vs. $24.4 \mathrm{~cm}$ diameter, a 1:8 ratio), whereas the study by Newman et al. (2001) used overall much smaller objects ( $1 \mathrm{~cm}$ vs. $6 \mathrm{~cm}$ diameter, a 1:6 ratio). Absolute size differences in the current study were intermediate $(5 \mathrm{~cm}$ vs. $18 \mathrm{~cm}$ diameter, a 1:3.6 ratio). It has been suggested that larger objects (non-graspable) are processed in the ventral visual-processing stream while smaller (graspable) objects are processed in the dorsal stream (Kaufman, Mareschal, \& Johnson, 2003). Early in development, successful discrimination of the graspability of two objects may be aided in young infants if the two objects activate different processing streams. In 5month-old infants, the small objects used by Newman et al. (2001) may have activated the dorsal processing stream, while our intermediate sized objects may have activated the ventral stream. Collectively, these results suggest that differentiation of objects based on graspability can be facilitated before the age of 5 months by using highly different sized objects. Perhaps providing these infants with additional reaching experiences (e.g., through 'sticky mittens'; Libertus \& Needham, 2010, 2011) could also facilitate the development of this preference for smaller, more easily graspable objects.

A key limitation of the current study is the small sample size, especially in the 4-5-monthold more-skilled reachers group. Due to the nature of our groups and the strong relation between age and reaching ability in infancy, having unequally group sizes was not avoidable in the current study. We hope to address this limitation in the future with larger sample and to explore the interesting group of 4-5-month-old more-skilled reachers in more detail.

\section{Conclusion}

Looking and reaching preferences for different sized objects are influenced by age and reaching status in 4-6-month old infants. As infants gain more proficiency with reaching actions, their responses to and engagement with objects changes. Four- to six-month-old infants with less developed reaching skills seem to choose objects primarily based on visual saliency without taking graspability into account. In contrast, 5-6-month-olds with more developed reaching skills seem to be at a transition point in development and begin to show 
more interest in objects that afford grasping. Although visual saliency strongly influences infants' initial orienting behavior at both ages tested here, 5-6-month-olds with more developed reaching skills show a second selection-for-action process which follows the initial orienting phase and seems to take graspability into account to guide both visual and manual preferences.

\section{Acknowledgments}

The work reported in this paper was completed as part of an undergraduate honors project by NZH. We would like to thank the parents and infants who generously spent their time participating in this study, and the undergraduate research assistants for their help with data collection and reduction. Financial support was provided by NIH grant R01 HD057120 to AN.

\section{References}

Adolph KE, Avolio AM. Walking infants adapt locomotion to changing body dimensions. Journal of Experimental Psychology: Human Perception and Performance. 2000; 26(3):1148-1166. [PubMed: 10884014]

Bertenthal, BI.; Clifton, RK. Perception and action. In: Kuhn, D.; Siegler, RS., editors. Handbook of child psychology: Vol. 2. Cognition, perception, and language. 5th ed.. New York: Wiley; 1998. p. 51-102.

Bourgeois KS, Khawar AW, Neal SA, Lockman JJ. Infant manual exploration of objects, surfaces, and their interrelations. Infancy. 2005; 8(3):233-252.

Brennan WM, Ames EW, Moore RW. Age differences in infants' attention to patterns of different complexities. Science. 1966; 151(3708):354-356. [PubMed: 5903347]

Bruner JS, Koslowski B. Visually preadapted constituents of manipulatory action. Perception. 1972; 1(1):3-14. [PubMed: 4680923]

Bushnell EW. The decline of visually guided reaching during infancy. Infant Behavior and Development. 1985; 8(2):139-155.

Bushnell EW, Boudreau JP. Motor development and the mind - the potential role of motor abilities as a determinant of aspects of perceptual development. Child Development. 1993; 64(4):1005-1021. [PubMed: 8404253]

Campos JJ, Witherington D, Anderson DI, Frankel CI, Uchiyama I, Barbu-Roth MA. Rediscovering development in infancy. Child Development. 2008; 79(6):1625-1632. [PubMed: 19037936]

Fagard J. Linked proximal and distal changes in the reaching behavior of 5- to 12-month-old human infants grasping objects of different sizes. Infant Behavior and Development. 2000; 23(3-4):317329.

Gibson EJ. Exploratory behavior in the development of perceiving, acting and acquiring of knowledge. Annual Review of Psychology. 1988; 39:1-41.

Hershenson M. Visual discrimination in the human newborn. Journal of Comparative and Physiological Psychology. 1964; 58:270-276. [PubMed: 14215402]

Hunter MA, Ames EW, Koopman R. Effects of stimulus complexity and familiarization time on infant preferences for novel and familiar stimuli. Developmental Psychology. 1983; 19(3):338-352.

Kaufman J, Mareschal D, Johnson MH. Graspability and object processing in infants. Infant Behavior and Development. 2003; 26(4):516-528.

Lederman SJ, Klatzky RL. Haptic perception: A tutorial. Attention Perception \& Psychophysics. 2009; 71(7):1439-1459.

Libertus, K. [Coding Software]. NC: Durham; 2008. StopFrameCoder (Version 0.9).

Libertus K, Needham A. Teach to reach: The effects of active vs. passive reaching experiences on action and perception. Vision Research. 2010; 50(24):2750-2757. [PubMed: 20828580]

Libertus K, Needham A. Reaching experience increases face preference in3-month-old infants. Developmental Science. 2011; 14(6):1355-1364. [PubMed: 22010895] 
Lobo MA, Galloway JC. Postural and object-oriented experiences advance early reaching, object exploration, and means - end behavior. Child Development. 2008; 79(6):1869-1890. [PubMed: 19037955]

Lobo MA, Galloway JC, Savelsbergh GJP. General and task-related experiences affect early object interaction. Child Development. 2004; 75(4):1268-1281. [PubMed: 15260877]

Lockman JJ, Ashmead DH, Bushnell EW. The development of anticipatory hand orientation during infancy. Journal of Experimental Child Psychology. 1984; 37(1):176-186. [PubMed: 6707575]

Needham A. Improvements in object exploration skills may facilitate the development of object segregation in early infancy. Journal of Cognition and Development. 2000; 1(2):131-156.

Newell KM, Scully DM, McDonald PV, Baillargeon R. Task constraints and infant grip configurations. Developmental Psychobiology. 1989; 22(8):817-831. [PubMed: 2636204]

Newman C, Atkinson J, Braddick O. The development of reaching and looking preferences in infants to objects of different sizes. Developmental Psychology. 2001; 37(4):561-572. [PubMed: $11444491]$

Pomerleau A, Malcuit G. Development of cardiac and behavioral responses to a 3-dimensional toy stimulation in one-month to 6-month-old infants. Child Development. 1980; 51(4):1187-1196. [PubMed: 7471922]

Pressley M, Borkowski JG, O’Sullivan JT. Memory strategy instruction is made of this: Metamemory and durable strategy use. Educational Psychologist. 1984; 19(2):94-107.

Rakison DH, Krogh L. Does causal action facilitate causal perception in infants youngerthan 6 months of age? Developmental Science. 2012; 15(1):43-53. [PubMed: 22251291]

Rocha NA, Silva FP, Tudella E. The impact of object size and rigidity on infant reaching. Infant Behavior and Development. 2006; 29(2):251-261. [PubMed: 17138280]

Rochat P, Goubet N. Development of sitting and reaching in 5-month-old to 6-month-old infants. Infant Behavior and Development. 1995; 18(1):53-68.

Rochat P, Goubet N, Senders SJ. To reach or not to reach? Perception of body effectivities by young infants. Infant and Child Development. 1999; 8(3):129-148.

Schwarzer G, Freitag C, Buckel R, Lofruthe A. Crawling is associated with mental rotation ability by 9-month-old infants. Infancy. 2013 http://dx.doi.org/10.1111/j.p1532-7078.2012.00132.x.

Slater A, Mattock A, Brown E. Size constancy at birth - newborn-infants responses to retinal and real size. Journal of Experimental Child Psychology. 1990; 49(2):314-322. [PubMed: 2332727]

von Hofsten C. Eye-hand coordination in the newborn. Developmental Psychology. 1982; 18(3):450 461.

von Hofsten C, Ronnqvist L. Preparation for grasping an object - a developmental-study. Journal of Experimental Psychology-Human Perception and Performance. 1988; 14(4):610-621. [PubMed: 2974872]

Yonas A, Elieff CA, Arterberry ME. Emergence of sensitivity to pictorial depth cues: Charting development in individual infants. Infant Behavior and Development. 2002; 25(4):495-514.

Yonas A, Hartman B. Perceiving the affordance of contact in four- and five-month-old infants. Child Development. 1993; 64(1):298-308. [PubMed: 8436036] 

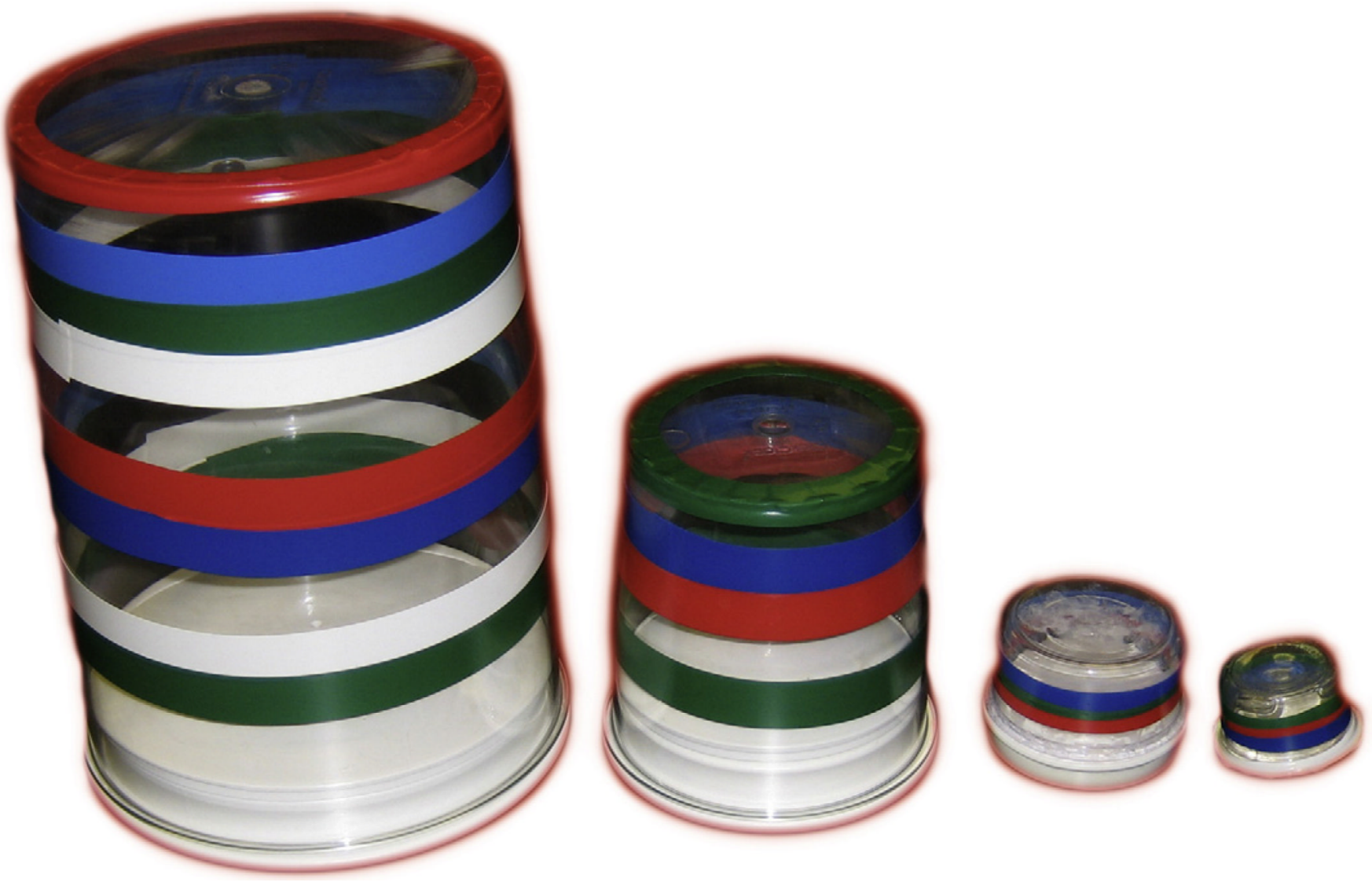

Fig. 1.

Four clear plastic cylinders of different size were used as stimuli. Object size ranged from 5 $\mathrm{cm}$ (object $1 \mathrm{on}$ far right), to $18 \mathrm{~cm}$ in diameter (object 4 on far left). 


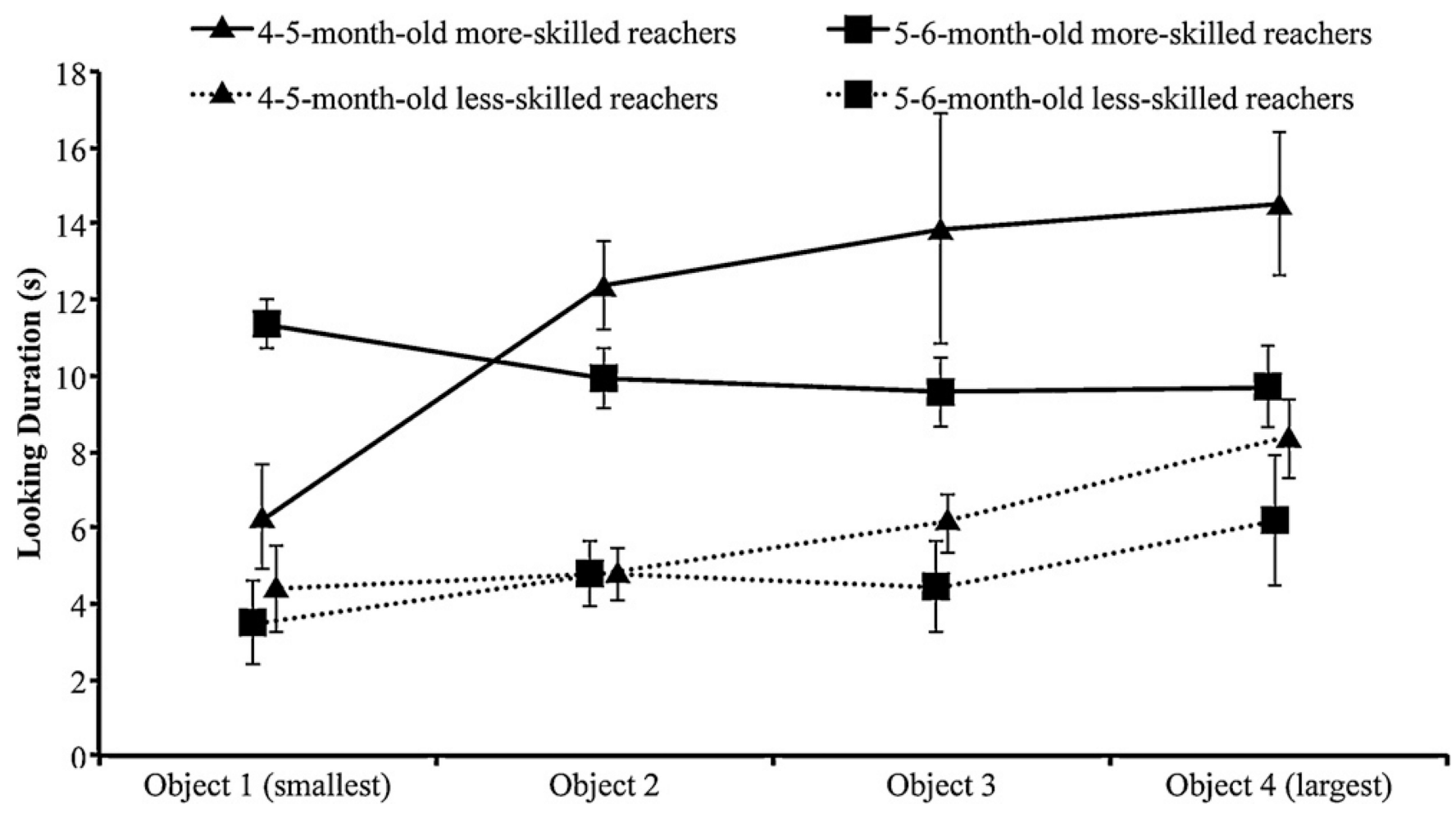

Fig. 2.

Infants' average looking duration for each of the 4 objects separated by age and reaching status. Error bars are SEM. 


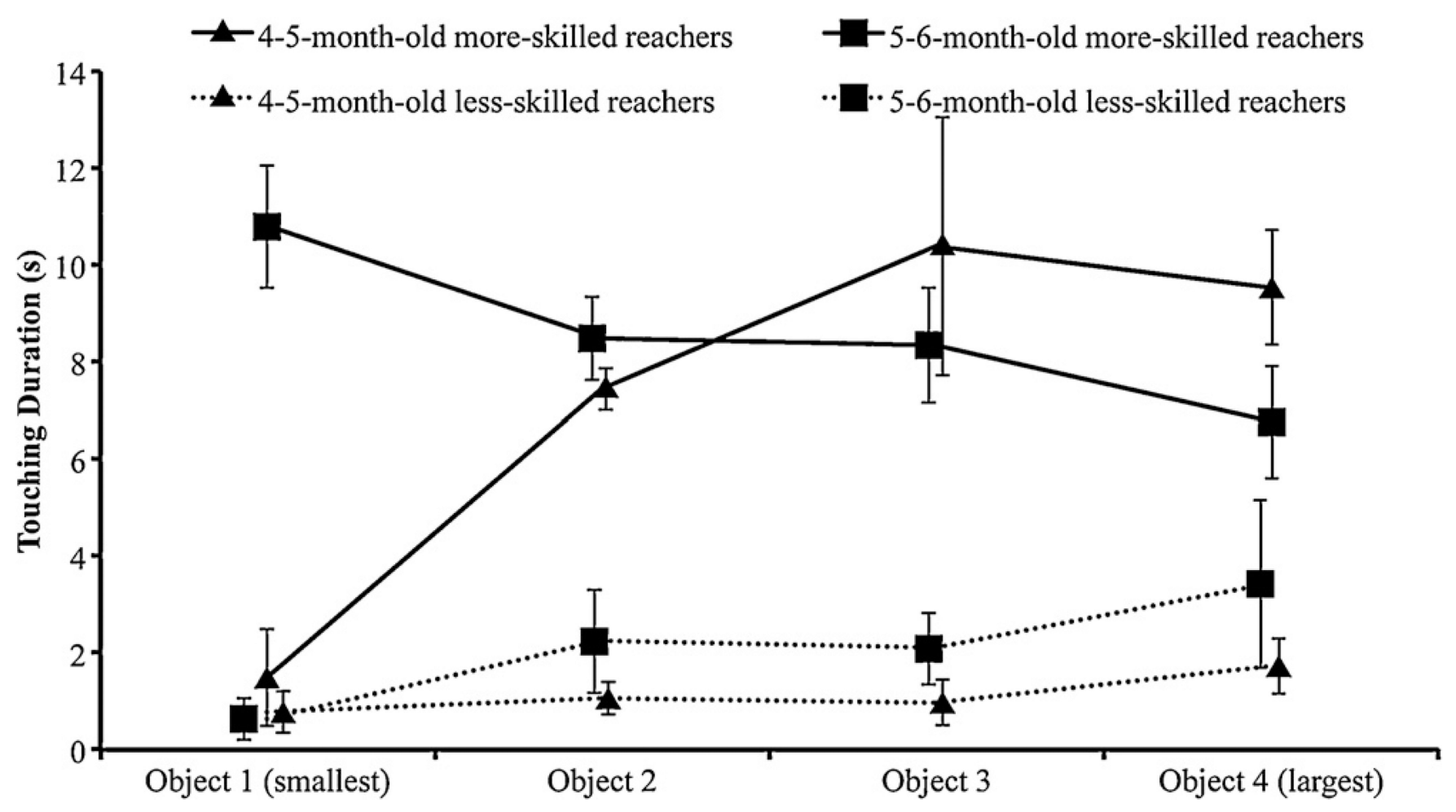

Fig. 3.

Infants' average touching duration for each of the 4 objects separated by age and reaching status. Error bars are SEM. 